\title{
LEVEL SETS OF THE FUNDAMENTAL SOLUTION AND HARNACK INEQUALITY FOR DEGENERATE EQUATIONS OF KOLMOGOROV TYPE
}

\author{
NICOLA GAROFALO AND ERMANNO LANCONELLI
}

\begin{abstract}
In this paper we establish a uniform Harnack inequality for a class of degenerate equations whose prototype is Kolmogorov's equations in $\mathbf{R}^{3}$ : $D_{y y} u-y D_{z} u-D_{t} u=0$. Our approach is based on mean value formulas for solutions of the equation under consideration on the level sets of the fundamental solution.
\end{abstract}

\section{INTRODUCTION}

In its simplest form Kolmogorov's equation can be written as

$$
D_{y y} u-y D_{z} u-D_{t} u=0, \quad(y, z, t) \in \mathbf{R}^{3} .
$$

The absence of the term $D_{z z} u$ in (1.1) makes the equation very degenerate. In [K] Kolmogorov showed that under certain conditions the probability density of a system with $2 n$ degrees of freedom satisfies a pde of the type

$$
\sum_{i, j=1}^{n} a_{i j} D_{y_{i} y_{j}} u-\sum_{i=1}^{n}\left(y_{i} D_{z_{i}} u+a_{i} D_{y_{i}} u\right)+a u-D_{t} u=0 \text {, }
$$

where the matrix $\left(a_{i j}\right)$ is symmetric and nondegenerate and $a_{i j}, a_{i}, a$ are functions of the variables $(y, z, t) \in \mathbf{R}^{n} \times \mathbf{R}^{n} \times \mathbf{R}$, see also [SV] and the classical monography [C]. Kolmogorov constructed an explicit fundamental solution of (1.1) smooth off the diagonal, thus proving that (1.1) is hypoelliptic. Weber [W] in 1951 and $\mathrm{Il}^{\prime}$ in [I] in 1964 proved the existence of a fundamental solution for (1.2) by Levi's method of the parametrix.

Kolmogorov's equation belongs to the class of Hörmander's operators $[\mathrm{H}]$

$$
L=\sum_{j=1}^{p} X_{j}^{2}-X_{0}
$$

where $X_{0}, X_{1}, \ldots, X_{p}$ are smooth first-order differential operators in $\mathbf{R}^{n+1}$ verifying the condition: The rank of the Lie algebra generated by $X_{0}, X_{1}, \ldots$,

Received by the editors November 18, 1988 .

1980 Mathematics Subject Classification (1985 Revision). Primary 31B10, 35C15, 35Q20; Secondary $35 \mathrm{~K} 65$.

The first author was supported by the National Science Foundation, grant DMS-8905338. 
$X_{p}$ is $n+1$ at every point. (1.1) is a special case of (1.3) corresponding to the choice $n=2, p=1, X_{1}=D_{y}, X_{0}=y D_{z}+D_{t}$. If $L$ in $(1.3)$ is

$$
L=\sum_{j=1}^{p} X_{j}^{2}-D_{t} \quad \text { in } \mathbf{R}^{n+1}
$$

and if $X_{1}, \ldots, X_{p}$ depend only on the space variable $x \in \mathbf{R}^{n}$, the fundamental solution $\Gamma(x, \xi, t)=\Gamma(x, t ; \xi, 0)$ with pole at $(\xi, 0)$ has been shown to satisfy the estimate

$$
\frac{1}{M\left|B_{d}(x, \sqrt{t})\right|} \exp \left[-\frac{M d(x, \xi)^{2}}{t}\right] \leq \Gamma(x, \xi, t) \leq \frac{M}{\left|B_{d}(x, \sqrt{t})\right|} \exp \left[-\frac{d(x, \xi)^{2}}{M t}\right] \text {, }
$$

when $t>0$, while $\Gamma(x, \xi, t)=0$ for $t \leq 0$, see [S, JS, and KS1]. In (1.5) $d(x, \xi)$ denotes the $\left\{X_{1}, \ldots, X_{p}\right\}$-control distance between $x$ and $\xi$, $B_{d}(x, \sqrt{t})=\{\xi \mid d(x, \xi)<\sqrt{t}\},\left|B_{d}(x, \sqrt{t})\right|=n$-dimensional Lebesgue measure of $B_{d}(x, \sqrt{t})$, and $M>0$ is a constant depending on $X_{1}, \ldots, X_{p}$. In [KS1, KS2] using (1.5) (which is established by suitable adapting some ideas of Nash [N], already employed by Fabes and Stroock in [FS]), the authors proved a uniform Harnack inequality of parabolic type of (1.4). By this we mean that the standard parabolic geometry $B\left(x_{0}, \sqrt{r}\right) \times\left(t_{0}-r, t_{0}\right)$, where $B\left(x_{0}, \sqrt{r}\right)=\left\{x|| x-x_{0} \mid<\sqrt{r}\right\}$, is in [KS1, KS2] replaced by the geometry $B_{d}\left(x_{0}, \sqrt{r}\right) \times\left(t_{0}-r, t_{0}\right)$, where $B_{d}$ is the $d$-ball introduced above.

On the one hand, equation (1.1) displays a parabolic feature in that its fundamental solution with pole at $(\eta, \zeta, 0) \in \mathbf{R}^{3}$

$$
\begin{cases}(\sqrt{3} / 2 \pi) t^{-2} \exp \left[-(y-\eta)^{2} / 4 t-\left(3 / t^{3}\right)(z-\zeta-(t / 2)(y+\eta))^{2}\right], & t>0, \\ 0, & t \leq 0,\end{cases}
$$

is supported in the half-space $\{t>0\}$. On the other hand, the variable $(y, z)$ do not play a distinguished role as do the space variables in (1.4).

In order to further emphasize the different nature of (1.1) with respect to (1.4) we show, by means of counterexample, that a parabolic type Harnack inequality may not be expected for nonnegative solution of (1.1). If the latter was true, in fact, then fixing a point $Q=(\bar{y}, 0,0) \in \mathbf{R}^{3}$, for every neighborhood $W$ of $Q$ we would have for $P_{t}=(\bar{y}, 0, t), t<0$,

$$
\varlimsup_{|t| \rightarrow 0}\left(\sup _{u \in H^{+}(W)} \frac{u\left(P_{t}\right)}{u(Q)}\right)<+\infty,
$$

where $H^{+}(W)$ denotes the family of all nonnegative solutions of (1.1) in $W$. The following example shows that, in general, this is not possible. We fix a point $\left(y_{0}, z_{0}, t_{0}\right) \in \mathbf{R}^{3}$, with $t_{0}<0$, and for $(y, z, t) \in \mathbf{R}^{3}$ let

$$
u(y, z, t)=\left\{\begin{array}{l}
\left(t-t_{0}\right)^{-2} \exp \left[-\frac{\left(y-y_{0}\right)^{2}}{4\left(t-t_{0}\right)}-\frac{3}{\left(t-t_{0}\right)^{3}}\left(z-z_{0}-\frac{t-t_{0}}{2}\left(y+y_{0}\right)\right)^{2}\right], t>t_{0} \\
0, \quad t \leq t_{0} .
\end{array}\right.
$$


This function is a nonnegative solution of equation (1.1) in the open set $\mathbf{R}^{3} \backslash\left\{\left(y_{0}, z_{0}, t_{0}\right)\right\}$. With $Q$ as above we let $P=\left(\bar{y}, 0, \theta t_{0}\right)$, where now $\bar{y} \neq 0$ and $\theta \in(0,1)$. Defining

$$
\lambda=\frac{z_{0}}{\left|t_{0}\right|}, \quad a=\frac{\bar{y}-y_{0}}{2}, \quad b=\frac{\bar{y}+y_{0}}{2},
$$

we obtain

$$
\frac{u(P)}{u(Q)}=\frac{1}{\theta^{2}} \exp \left\{-\frac{3}{\left|t_{0}\right|}\left(\frac{1}{\theta}-1\right)\left[\left(\left(\frac{1}{\theta}+1\right) \lambda+b\right)^{2}+\frac{a^{2}}{3}-\frac{\lambda^{2}}{\theta}\right]\right\} .
$$

If we now choose $y_{0}$ and $z_{0}$ in (1.9) such that

$$
\left(\frac{1}{\theta}+1\right) \lambda+b=0, \quad \frac{a^{2}}{3}-\frac{\lambda^{2}}{\theta}<0,
$$

we obtain from (1.10)

$$
\lim _{\left|t_{0}\right| \rightarrow 0} \frac{u(P)}{u(Q)}=+\infty
$$

This contradicts (1.7). We note explicitly that (1.11) reads

$$
\left(1+\frac{1}{\theta}\right) \frac{z_{0}}{\left|t_{0}\right|}=-\frac{\bar{y}+y_{0}}{2}, \quad\left|\bar{y}-y_{0}\right|<\frac{\sqrt{3 \theta}}{1+\theta}\left|\bar{y}+y_{0}\right| .
$$

We remark that it is possible to choose $y_{0} \in \mathbf{R}$ such that the second part of (1.12) holds iff $\bar{y} \neq 0$, and that for any choice of $y_{0}, z_{0}$ as in (1.12), the definition (1.8) gives a one-parameter family of solutions $u=u_{t_{0}}$ of (1.1) in a suitably fixed neighborhood of $Q$.

This paper originates from an attempt to establish a uniform Harnack inequality for a class of degenerate equations containing (1.1) and (1.2). Our approach is inspired to the elementary proof of Harnack inequality for parabolic equations we gave in [GL2]. The underlying idea of our method is that the exact geometry of a uniform Harnack inequality is determined by the level sets of the fundamental solution. For equations as (1.1) or (1.2) this geometry is rather complicated, and the "variable coefficient" case (1.2) is not, from an intrinsic viewpoint, much more difficult than the "constant coefficient" case (1.1). For this reason, in this paper we confine ourselves to the study of a class of equations which contains (1.2), when in the latter $\left(a_{i j}\right)$ is a constant matrix, and $a_{i}=a=0$. We hope to come back, in a future study, to the analysis of the general case. In what follows we let $n, k, l$ be nonnegative integers such that $n=k+l$. We denote points $x \in \mathbf{R}^{n}$ by $x=\left(\begin{array}{c}y \\ z\end{array}\right)^{T}$, where $y \in \mathbf{R}^{k}, z \in \mathbf{R}^{l}$, and consider the following class of equations

$$
L u=\operatorname{div}_{y}\left(A \nabla_{y} u\right)-B y \cdot \nabla_{z} u-D_{t} u=0 \quad \text { in } \mathbf{R}^{n+1} .
$$

In (1.13) $A=\left(a_{i j}\right)$ is a $k \times k$ matrix, $B=\left(b_{m j}\right), m=1, \ldots, l, j=1, \ldots, k$, is a $l \times k$ matrix such that

$$
\left\{\begin{array}{l}
A \text { is symmetric and positive definite, } \\
C=\left(B A B^{T}\right)^{-1} \text { exists and is a } l \times l \text { positive definite matrix. }
\end{array}\right.
$$


Points $y \in \mathbf{R}^{k}, z \in \mathbf{R}^{l}$ are thought of as column vectors, so that $B y$ is the $l \times 1$ column vector obtained by usual matrix multiplication. For a given matrix $M, M^{T}$ denotes its transpose. $\nabla_{y} u$ and $\nabla_{z} u$ respectively denote the column vectors $\left(D_{y_{1}} u, \ldots, D_{y_{k}}\right)^{T},\left(D_{z_{1}} u, \ldots, D_{z_{t}} u\right)^{T}$.

Under the assumptions (1.14) the operator $L$ in (1.13) satisfies Hörmander's condition cited above on the rank of the Lie algebra. An explicit fundamental solution for (1.13) has been constructed in $[\mathrm{H}]$, see also $[\mathrm{K}]$,

$$
\begin{aligned}
\Gamma(x, t ; \xi, \tau) & =\Gamma(y, z, t ; \eta, \zeta, \tau) \\
& =\left\{\begin{array}{l}
\chi(t-\tau)^{-\gamma} \exp \left[-\frac{|y-\eta|_{A}^{2}}{(t-\tau)}-\frac{3}{(t-\tau)^{3}}\left|z-\zeta-\frac{(t-\tau)}{2} B(y+\eta)\right|_{C}^{2}\right], \\
0, \quad t \leq \tau .
\end{array} \quad t>\tau,\right.
\end{aligned}
$$

In (1.15) we have respectively denoted by $|\cdot|_{A}^{2}$ and $|\cdot|_{C}^{2}$ the quadratic forms

$$
|y|_{A}^{2}=A^{-1} y \cdot y, \quad y \in \mathbf{R}^{k}, \quad|z|_{C}^{2}=C z \cdot z, \quad z \in \mathbf{R}^{\prime},
$$

whereas we have set

$$
\gamma=\frac{k+3 l}{2}, \quad \chi=\pi^{-n / 2} \frac{3^{l / 2}}{2^{k}}\left(\frac{\operatorname{det} C}{\operatorname{det} A}\right)^{1 / 2} .
$$

Our proof of Harnack inequality for equation (1.13) is rather geometric in spirit. It is based on mean value formulas and a property of the level sets of the fundamental solution $\Gamma$ in (1.15) which is reminiscent of the following elementary property of Euclidean balls: Given a ball $B\left(x_{0}, r\right), x_{0} \in \mathbf{R}^{n}, r>0$, for any $x \in B\left(x_{0}, r\right)$ and any $\delta \in(0,1)$ the ball $B(x, \delta r)$ is completely contained in $B\left(x_{0}, 2 r\right)$. This property, which is a simple consequence of the triangle inequality, plays a crucial role in the classical proof of Harnack inequality for harmonic functions via mean value formulas. In $\S 2$ we prove that an ad hoc version is true for the level sets of $\Gamma$ in (1.15).

In order to be more specific we need to introduce some notation. Let $(x, t) \in$ $\mathbf{R}^{n+1}$, for $r>0$ we set

$$
\Omega_{r}(x, t)=\left\{(\xi, \tau) \in \mathbf{R}^{n+1} \mid \Gamma(x, t ; \xi, \tau)>\chi / r^{\gamma}\right\},
$$

where $\gamma$ and $\chi$ are as in (1.17). By analogy with the Euclidean case we call the set $\Omega_{r}(x, t)$ the Kolmogorov ball "centered" at $(x, t)$ with radius $r$. The geometry of the set $\Omega_{r}(x, t)$ changes with the position of the center $(x, t)$, and may not be immediately obvious to the reader. It may be helpful to look at the following equivalent description

$$
\begin{aligned}
\Omega_{r}(x, t)=\left\{(\xi, \tau) \in \mathbf{R}^{n+1}\left|\frac{|y-\eta|_{A}^{2}}{4}+\frac{3}{(t-\tau)^{2}}\right| z\right. & -\zeta-\left.\frac{(t-\tau)}{2} B(y+\eta)\right|_{C} ^{2} \\
& \left.<R_{r}(t-\tau), t-r<\tau<t\right\},
\end{aligned}
$$


where we have set $R_{r}(t-\tau)=\gamma(t-\tau) \ln (r /(t-\tau))$. From (1.19) it is clear that the sections of $\Omega_{r}(x, t)$ with hyperplanes $\tau=t-s, 0<s<r$, are ellipsoids centered at the point $(y, z-s B y, t-s)$. As $s$ runs between 0 and $r$, this point describes a segment of straight line in $\mathbf{R}^{n+1}$ which is parallel to the time axis only when $y=0$. The geometric property of the level sets of $\Gamma$ referred to above is the following:

Three-Balls Lemma. Let $\left(x_{0}, t_{0}\right) \in \mathbf{R}^{n+1}, r>0$ be fixed. Then for any $\varepsilon \in$ $(0,1)$, there exists $\delta=\delta(\varepsilon)<1$ such that for every $(x, t) \in \Omega_{r}\left(x_{0}, t_{0}\right)$, with $t_{0}-t \geq \varepsilon r$, we have

$$
\Omega_{\delta r}(x, t) \subset \Omega_{2 r}\left(x_{0}, t_{0}\right) .
$$

We emphasize that the time-lag $t_{0}-t \geq \varepsilon r$ in the above lemma is crucial to obtain (1.20). This reflects the fact that the fundamental solution of $L$ in (1.13) is supported in a half-space. We prove the three-balls lemma in $\S 2$.

In $\S 3$ we establish some representation formulas on the Kolmogorov balls for smooth functions in $\mathbf{R}^{n+1}$. These formulas generalize to the present degenerate setting previous results established in [GL1, GL2] for parabolic equations. In the particular case in which the smooth function is a solution of $(1.13)$ we obtain the following mean value property:

$$
u\left(x_{0}, t_{0}\right)=\int_{\Omega_{r}\left(x_{0}, t_{0}\right)} u(x, t) E_{r}\left(x_{0}, t_{0} ; x, t\right) d x d t,
$$

where

$$
E_{r}\left(x_{0}, t_{0} ; x, t\right)=\frac{\chi}{r^{\gamma}} \frac{A\left(\nabla_{y} \Gamma\left(x_{0}, t_{0} ; x, t\right)\right) \cdot \nabla_{y} \Gamma\left(x_{0}, t_{0} ; x, t\right)}{\Gamma^{2}\left(x_{0}, t_{0} ; x, t\right)}
$$

We stress the absence of the $\nabla_{z}$-part of the gradient of $\Gamma$ in (1.22). We mention that a surface mean-value formula for solutions of (1.13) had been obtained by Kupcov in $[\mathrm{Ku}]$. Such a formula allowed him to prove a principle or propagation of maxima, yet it cannot be directly used to obtain Harnack inequality. In spite of its aesthetical appeal (1.21) cannot be used either, the reason being the unboundedness of the kernel appearing in it. (1.21) does, however, contain a redeeming feature, which can be illustated as follows. If $u \in C \quad\left\{\mathbf{R}^{n+1}\right\}$ is a solution of (1.13), then for any $h \in N, u$ is also a solution in $\mathbf{R}^{n+h+1}$ of the equation

$$
L_{h} u=L u+\Delta_{h} u=0,
$$

where $L$ is as in (1.13) and $\Delta_{h}$ denotes the Laplacian acting on $w \in \mathbf{R}^{h}$. But $L_{h}$ is an operator in $\mathbf{R}^{n+h+1}$ which belongs to the same class of $L$ in (1.13). Therefore, $u$ possesses in $\mathbf{R}^{n+h+1}$ a mean value property on the level sets of the fundamental solution $\Gamma_{h}$ of $L_{h}$ similar to (1.21). As we will prove in $\S 3$ in this new mean value formula, the fictitious variable $w$ can be eliminated, 


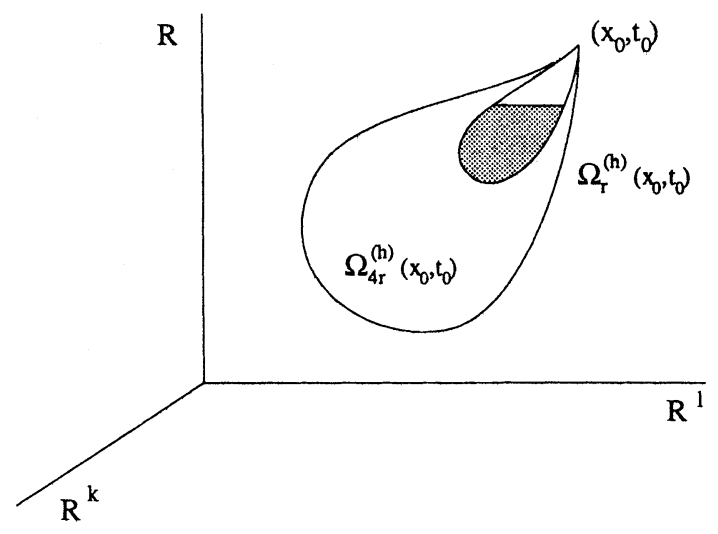

FIGURE 1

thus yielding

$$
u\left(x_{0}, t_{0}\right)=\int_{\Omega_{r}^{(h)}\left(x_{0}, t_{0}\right)} u(x, t) E_{r}^{(h)}\left(x_{0}, t_{0} ; x, t\right) d x d t,
$$

where for every $h \in \mathbf{N}$ we have set

$$
\Omega_{r}^{(h)}\left(x_{0}, t_{0}\right)=\left\{(x, t) \in \mathbf{R}^{n+1} \mid\left(t_{0}-t\right)^{-h / 2} \Gamma\left(x_{0}, t_{0} ; x, t\right)>\chi h / r^{y_{h}}\right\},
$$

with

$$
\gamma_{h}=\gamma+h / 2, \quad \chi_{h}=(4 \pi)^{-h / 2} \chi,
$$

and $E_{r}^{(h)}\left(x_{0}, t_{0} ; x, t\right)$ is a suitable kernel, see (3.32). The nice feature of (1.24) is that the kernel $E_{r}^{(h)}$ becomes less and less singular as $h$ grows. In particular, $E_{r}^{(h)}$ is bounded above on the set $\Omega_{r}^{(h)}\left(x_{0}, t_{0}\right)$, provided that $h>2$. We remark that, as it will be apparent from the proof, the three-balls lemma holds unchanged if in its statement we replace the Kolmogorov balls $\Omega_{r}$ with the modified balls $\Omega_{r}^{(h)}$.

In $\S 4$ using (1.24) and the three-balls lemma we prove the following:

Theorem 1.1 (Harnack Inequality). Let $\left(x_{0}, t_{0}\right) \in \mathbf{R}^{n+1}, r>0$, and $u$ be $a$ nonnegative solution of (1.13) in $\Omega_{4 r}^{(h)}\left(x_{0}, t_{0}\right)$, with $h \in \mathbf{N}, h>2$ fixed. For any $\varepsilon \in(0,1)$ there exists a positive constant $C=C(\varepsilon, n, h, L)$ such that

$$
\sup _{\substack{(x, t) \in \Omega^{(h)}\left(x_{0}, t_{0}\right) \\ t_{0}-t \geq \varepsilon r}} u(x, t) \leq C u\left(x_{0}, t_{0}\right) .
$$

The geometry is illustrated in Figure 1.

We close this section with an application of Theorem 1.1 to a notable class of degenerate equations. In $\mathbf{R}^{n}$ we consider the equation

$$
E u=\operatorname{div}_{y}\left(A \nabla_{y} u\right)-B y \cdot \nabla_{z} u=0,
$$


where the matrices $A$ and $B$ are as in (1.13), (1.14). The operator $E$ in (1.28) is again a hypoelliptic operator of Hörmander's type. An interesting particular instance of (1.28) is given by the case $l=1$, so that $n=k+1$. Then $B$ is a $k$-dimensional row vector and (1.28) becomes

$$
E u=\operatorname{div}_{y}\left(A \nabla_{y} u\right)-B y D_{z} u=0 \quad \text { in } \mathbf{R}^{k+1} .
$$

(1.29) is a degenerate equation changing type along the hyperplane $H=\{(y, z)$ $\left.\in \mathbf{R}^{k+1} \mid B y=0\right\}$. It is forward parabolic in the half-space $H^{+}=\{(y, z) \in$ $\left.\mathbf{R}^{k+1} \mid B y>0\right\}$, backward parabolic in the half-space $H^{-}=\mathbf{R}^{k+1} \backslash \overline{H^{+}}$. Using Theorem 1.1 and the obvious observation that any solution of (1.28) can be thought of as a time-independent solution of (1.13), we obtain a uniform Harnack inequality for (1.28). In order to formulate it we introduce some notation. For $x_{0}=\left(y_{0}, z_{0}\right) \in \mathbf{R}^{n}, r>0, h>2$ fixed and $\varepsilon \in(0,1)$ we define

$$
\begin{aligned}
B_{r}^{\varepsilon}\left(x_{0}\right)=\bigcup_{\varepsilon r<s<r}\left\{(y, z) \in \mathbf{R}^{n}\left|\frac{1}{4}\right| y-\left.y_{0}\right|_{A} ^{2}+\frac{3}{s^{2}} \mid z-z_{0}\right. & \\
& \left.+\frac{s}{2} B\left|\left(y+y_{0}\right)\right|_{C}^{2}<\gamma_{h} s \ln \left(\frac{r}{s}\right)\right\},
\end{aligned}
$$

where ||$_{A},||_{C}$ are as in (1.16), and $\gamma_{h}$ is as in (1.26). We agree to let $B_{r}^{0}\left(x_{0}\right)=B_{r}\left(x_{0}\right)$.

Theorem 1.2. Let $x_{0} \in \mathbf{R}^{n+1}, r>0$, and let $u \geq 0$ be a solution of (1.28) in $B_{4 r}\left(z_{0}\right)$. Then for any $\varepsilon \in(0,1)$ there exists a positive number $C=$ $C(\varepsilon, n, h, E)$ such that

$$
\sup _{B_{r}^{\varepsilon}\left(x_{0}\right)} u \leq C u\left(x_{0}\right) \text {. }
$$

We note explicitly that, as previously noted, for every $s, \varepsilon r<s<r$, the set

$$
\left\{(y, z) \in \mathbf{R}^{n}\left|\frac{1}{4}\right| y-\left.y_{0}\right|_{A} ^{2}+\frac{3}{s^{2}}\left|z-z_{0}+\frac{s}{2} B\left(y+y_{0}\right)\right|_{C}^{2}<\gamma_{h} s \ln \left(\frac{r}{s}\right)\right\}
$$

is an ellipsoid centered at the point $\left(y_{0}, z_{0}+s B y_{0}\right)$. Therefore, if $y_{0}=0$ the center of the ellipsoid is no longer varying with $s$, and, in fact, coincides with $x_{0}=\left(y_{0}, z_{0}\right)=\left(0, z_{0}\right)$. As a consequence of these considerations, we see that when $x_{0}=\left(0, z_{0}\right)$ Theorem 1.2 yields an elliptic-type Harnack inequality.

\section{Proof of the geometric lemma}

In this section we prove the three-balls lemma stated in the introduction. In what follows we use the notation introduced in (1.19). The letters $x, x_{0}, \xi$ will respectively denote the points $(y, z),\left(y_{0}, z_{0}\right),(\eta, \zeta)$ in $\mathbf{R}^{n}$. Recalling (1.19) we see that the condition $(x, t) \in \Omega_{r}\left(x_{0}, t_{0}\right)$ can be written

$$
\begin{aligned}
& \frac{1}{4}\left|y-y_{0}\right|_{A}^{2}+\frac{3}{\left(t_{0}-t\right)^{2}}\left|z-z_{0}+\frac{\left(t_{0}-t\right)}{2} B\left(y-y_{0}\right)\right|_{C}^{2}<R_{r}\left(t_{0}-t\right) \\
& 0<t_{0}-t<r .
\end{aligned}
$$


We pick a point $(\xi, \tau) \in \Omega_{\delta r}(x, t)$. This means

$$
\frac{1}{4}|\eta-y|_{A}^{2}+\frac{3}{(t-\tau)^{2}}\left|\zeta-z+\frac{(t-\tau)}{2} B(y+\eta)\right|_{C}^{2}<R_{\delta r}(t-\tau), \quad 0<t-\tau<\delta r .
$$

We want to prove that for every $\varepsilon \in(0,1)$ there exists $\delta=\delta(\varepsilon)$ such that if $t_{0}-t \geq \varepsilon r$, then (1.20) holds, i.e.,

$$
\frac{1}{4}\left|\eta-y_{0}\right|_{A}^{2}+\frac{3}{\left(t_{0}-\tau\right)^{2}}\left|\zeta-z_{0} \frac{\left(t_{0}-\tau\right)}{2} B\left(y_{0}+\eta\right)\right|_{C}^{2}<R_{2 r}\left(t_{0}-\tau\right) .
$$

Now we set

$$
u=\frac{t_{0}-t}{r}, \quad v=\frac{t-\tau}{r}
$$

so that from (2.1), (2.2) we have $0<u<1,0<v<\delta$. Moreover, since we want $t_{0}-t \geq \varepsilon r$, we have the further limitation $\varepsilon \leq u<1$. Letting

$$
\begin{array}{lll}
\bar{y}=r^{-1 / 2} y, & \bar{y}_{0}=r^{-1 / 2} y_{0}, & \bar{\eta}=r^{-1 / 2} \eta, \\
\bar{z}=r^{-3 / 2} z, & \bar{z}_{0}=r^{-3 / 2} z_{0}, & \bar{\zeta}=r^{-3 / 2} \zeta,
\end{array}
$$

(2.1), (2.2), and (2.3) become

$(2.1)^{\prime} \frac{1}{4}\left|\bar{y}-\bar{y}_{0}\right|_{A}^{2}+\frac{3}{u^{2}}\left|\bar{z}-\bar{z}_{0}+\frac{u}{2} B\left(\bar{y}_{0}+\bar{y}\right)\right|_{C}^{2}<R_{1}(u), \quad \varepsilon \leq u<1$,

$(2.2)^{\prime} \quad \frac{1}{4}|\bar{\eta}-\bar{y}|_{A}^{2}+\frac{3}{v^{2}}\left|\bar{\zeta}-\bar{z}+\frac{v}{2} B(\bar{y}+\bar{\eta})\right|_{C}^{2}<R_{\delta}(v), \quad 0<v<\delta$,

$(2.3)^{\prime} \frac{1}{4}\left|\bar{\eta}-\bar{y}_{0}\right|_{A}^{2}+\frac{3}{(u+v)^{2}}\left|\bar{\zeta}-\bar{z}_{0}+\frac{(u+v)}{2} B\left(\bar{y}_{0}+\bar{\eta}\right)\right|_{C}^{2}<R_{2}(u+v)$.

Now we let

$$
\begin{cases}\bar{y}-\bar{y}_{0}=Y^{\prime}, & \bar{\eta}-\bar{y}=Y^{\prime \prime} \\ \bar{z}-\bar{z}_{0}+\frac{u}{2}\left(\bar{y}_{0}+\bar{y}\right)=Z^{\prime}, & \bar{\zeta}-\bar{z}+\frac{v}{2} B(\bar{y}+\bar{\eta})=Z^{\prime \prime}\end{cases}
$$

Simple algebraic manipulations give

$$
\bar{\zeta}-\bar{z}_{0}+\frac{(u+v)}{2} B\left(\bar{y}_{0}+\bar{\eta}\right)=Z^{\prime}+Z^{\prime \prime}+B\left(\frac{u}{2} Y^{\prime \prime}-\frac{v}{2} Y^{\prime}\right) .
$$

With the substitution $(2.4)$ conditions $(2.1)^{\prime}-(2.33)^{\prime}$ then become

$$
\begin{array}{ll}
\frac{1}{4}\left|Y^{\prime}\right|_{A}^{2}+\frac{3}{u^{2}}\left|Z^{\prime}\right|_{C}^{2}<R_{1}(u), & \varepsilon \leq u<1, \\
\frac{1}{4}\left|Y^{\prime \prime}\right|_{A}^{2}+\frac{3}{v^{2}}\left|Z^{\prime \prime}\right|_{C}^{2}<R_{\delta}(v), & 0<v<\delta,
\end{array}
$$

$$
\frac{1}{4}\left|Y^{\prime}+Y^{\prime \prime}\right|_{A}^{2}+\frac{3}{(u+v)^{2}}\left|Z^{\prime}+Z^{\prime \prime}+B\left(\frac{u}{2} Y^{\prime \prime}-\frac{v}{2} Y^{\prime}\right)\right|_{C}^{2}<R_{2}(u+v) .
$$


Recalling that $R_{\delta}(v)=\gamma v \ln (\delta / v)$ we see that letting $\delta \rightarrow 0^{+}$in $(2.2)^{\prime \prime}$ we obtain $Y^{\prime \prime} \rightarrow 0 \in R^{\dot{k}}$ and $Z^{\prime \prime} \rightarrow 0 \in R^{l}$. Therefore, when $\delta \rightarrow 0^{+}(2.3)^{\prime \prime}$ becomes

$$
\frac{1}{4}\left|Y^{\prime}\right|_{A}^{2}+\frac{3}{u^{2}}\left|Z^{\prime}\right|_{C}^{2}<R_{2}(u)
$$

and this inequality is true because of $(2.1)^{\prime \prime}$. Using these observations, by an easy compactness argument we obtain for any $\varepsilon \in(0,1)$ the existence of a $\delta=\delta(\varepsilon)>0$ such that $(2.3)^{\prime \prime}$ holds for all $\varepsilon \leq u<1,0 \leq v<\delta, Y^{\prime}, Y^{\prime \prime}$ for which $(2.1)^{\prime \prime},(2.2)^{\prime \prime}$ hold. But $(2.3)^{\prime \prime}$ is equivalent, via the above change of variables, to (2.3), and the latter in turn is (1.20). This completes the proof.

\section{Mean value formulas}

In this section we will prove (1.21), and, subsequently, (1.24). With $L$ as in (1.13) we denote by

$$
L^{*} v=\operatorname{div}_{y}\left(A \nabla_{y} v\right)+B y \cdot \nabla_{z} v+D_{t} v=0
$$

the adjoint equation of (1.13). Our starting point is the formula

$$
v L u-u L^{*} v=\operatorname{div}_{(x, t)}\left[\left(v A \nabla_{y} u-u A \nabla_{y} v, u v B y,-u v\right)\right],
$$

where we have denoted by $\operatorname{div}_{(x, t)}=\operatorname{div}_{(y, z, t)}$ the total divergence of the vector field within brackets. If $D \subset \mathbf{R}^{n+1}$ is a domain with smooth boundary and $u$, $v$ are smooth functions on $\bar{D}$, the divergence theorem and (3.1) yield

$\int_{D}\left(v L u-u L^{*} v\right) d x d t=\int_{\partial D}\left[\left(v A \nabla_{y} u-u A \nabla_{y} v\right) \cdot \vec{N}_{y}+u v B y \cdot \vec{N}_{z}-u v N_{t}\right] d H_{n}$,

where $\vec{N}=\left(\vec{N}_{y}, \vec{N}_{z}, N_{t}\right)$ is the outward normal to $D$ and $d H_{n}$ denotes $n$-dimensional Hausdorff measure. If $u$ is such that $L u=0$, then choosing $v \equiv 1$ in (3.2) we obtain

$$
\int_{\partial D}\left[A \nabla_{z} u \cdot \vec{N}_{y}+u B y \cdot \vec{N}_{z}-u N_{t}\right] d H_{n}=0 .
$$

If instead we only let $v \equiv 1$ in (3.2) we have

$$
\int_{\partial D}\left[A \nabla_{y} u \cdot \vec{N}_{y}+u B y \cdot \vec{N}_{z}-u N_{t}\right] d H_{n}=\int_{D} L u d x d t .
$$

Now we fix $\left(x_{0}, t_{0}\right)=\left(y_{0}, z_{0}, t_{0}\right) \in \mathbf{R}^{n+1}, r>0$ and consider the Kolmogorov ball $\Omega_{r}\left(x_{0}, t_{0}\right)$. For $\varepsilon \in(0, r)$ we let $D_{\varepsilon}=\Omega_{r}\left(x_{0}, t_{0}\right) \cap\left\{(x, t) \mid t<t_{0}-\varepsilon\right\}$. We set $v(x, t)=\Gamma\left(x_{0}, t_{0} ; x, t\right)$ with $\Gamma$ as in (1.15), and note that $L^{*} v=0$ in $D_{\varepsilon}$. We denote by $\psi_{r}\left(x_{0}, t_{0}\right)$ the Kolmogorov sphere centered at $\left(x_{0}, t_{0}\right)$ with radius $r$, i.e.,

$\psi_{r}\left(x_{0}, t_{0}\right)=\partial \Omega_{r}\left(x_{0}, t_{0}\right)=\left\{(\xi, \tau) \in \mathbf{R}^{n+1} \mid \Gamma\left(x_{0}, t_{0} ; \xi, \tau\right)=\chi / r^{\gamma}\right\} \cup\left\{\left(x_{0}, t_{0}\right)\right\}$, and for $\varepsilon$ as above we set $\psi_{\varepsilon}=\psi_{r}\left(x_{0}, t_{0}\right) \cap\left\{(x, t) \mid t<t_{0}-\varepsilon\right\}, I_{\varepsilon}=\bar{\Omega}_{r}\left(x_{0}, t_{0}\right) \cap$ $\left\{(x, t) \mid t=t_{0}-\varepsilon\right\}$. With this notation we have $\partial D_{\varepsilon}=\psi_{\varepsilon} \cup I_{\varepsilon}$. Moreover, since 
$\Gamma\left(x_{0}, t_{0} ; \cdot, \cdot\right) \in C^{\infty}\left(\mathbf{R}^{n+1} \backslash\left\{x_{0}, t_{0}\right\}\right)$, by Sard's theorem for a.e. $r>0$ the set $\psi_{\varepsilon}$ is a smooth $n$-dimensional manifold for every $\varepsilon \in(0, r)$. In the sequel we suppose that an $r>0$ has been fixed for which $\psi_{\varepsilon}$ is a smooth manifold. We let $u \in C^{\infty}\left(\mathbf{R}^{n+1}\right)$ and apply (3.2) to this function, to $v$ defined above and to the set $D_{\varepsilon}$, obtaining

$\int_{D_{\varepsilon}} \Gamma L u d x d t=\int_{\psi_{\varepsilon} \cup I_{\varepsilon}}\left\{\left[A \nabla_{y} u \cdot \vec{N}_{y}+u B y \cdot \vec{N}_{z}-u N_{t}\right] \Gamma-u A \nabla_{y} \Gamma \cdot \vec{N}_{y}\right\} d H_{n}$,

where in (3.6) we have set $\Gamma=\Gamma\left(x_{0}, t_{0} ; \cdot, \cdot\right)$. Since by (3.5) $\Gamma=\chi r^{-\gamma}$ on $\psi_{\varepsilon}$ and $N_{t}=1$ on $E_{\varepsilon}$, we obtain from (3.6)

$$
\begin{aligned}
\int_{D_{\varepsilon}} \Gamma L u d x d t= & \frac{\chi}{r^{\gamma}} \int_{\psi_{\varepsilon}}\left[A \nabla_{y} u \cdot \vec{N}_{y}+u B y \cdot \vec{N}_{z}-u N_{t}\right] d H_{n} \\
& -\int_{I_{\varepsilon}} u \Gamma d H_{n}-\int_{\psi_{\varepsilon}} u A \nabla_{y} \Gamma \cdot \vec{N}_{y} d H_{n} .
\end{aligned}
$$

Using the Dirac function property of $\Gamma$ we have

$$
\lim _{\varepsilon \rightarrow 0^{+}} \int_{I_{\varepsilon}} u \Gamma d H_{n}=u\left(x_{0}, t_{0}\right) .
$$

Letting then $\varepsilon \rightarrow 0^{+}$in (3.7) finally gives

$$
\begin{aligned}
\int_{\Omega_{r}\left(x_{0}, t_{0}\right)} \Gamma L u d x d t= & \frac{\chi}{r^{\gamma}} \int_{\psi_{r}\left(x_{0}, t_{0}\right)}\left[A \nabla_{y} u \cdot \vec{N}_{y}+u B y \cdot \vec{N}_{z}-u N_{t}\right] d H_{n} \\
& -u\left(x_{0}, t_{0}\right)-\int_{\psi_{r}\left(x_{0}, t_{0}\right)} u A \nabla_{y} \Gamma \cdot \vec{N}_{y} d H_{n} .
\end{aligned}
$$

In virtue of (3.4) we finally obtain from (3.8)

$$
-\int_{\psi_{r}\left(x_{0}, t_{0}\right)} u A \nabla_{y} \Gamma \cdot \vec{N}_{y} d H_{n}=u\left(x_{0}, t_{0}\right)+\int_{\Omega_{r}\left(x_{0}, t_{0}\right)} L u\left[\Gamma-\frac{\chi}{r^{\gamma}}\right] d x d t
$$

Formula (3.9) is our starting point. Changing $r$ in $\rho$ in (3.9), multiplying both sides by $\rho^{\gamma-1}$ and integrating in $\rho$ between 0 and $r$, recalling that on $\psi_{r}\left(x_{0}, t_{0}\right) \backslash\left\{\left(x_{0}, t_{0}\right)\right\}, \vec{N}_{y}=-\nabla_{y} \Gamma /\left|\left(\nabla_{y} \Gamma, \nabla_{z} \Gamma, D_{t} \Gamma\right)\right|$, we have

$$
\begin{aligned}
& \int_{0}^{r} \rho^{\gamma}\left(\int_{\Gamma=x / \rho^{\gamma}} u \frac{A \nabla_{y} \Gamma \cdot \nabla_{y} \Gamma}{\left|\nabla_{x, t} \Gamma\right|} d H_{n}\right) \frac{d \rho}{\rho} \\
& \quad=\frac{r^{\gamma}}{\gamma} u\left(x_{0}, t_{0}\right)+\int_{0}^{r} \rho^{\gamma-1}\left(\int_{\Omega_{\rho}\left(x_{0}, t_{0}\right)} L u\left[\Gamma-\frac{\chi}{\rho^{\gamma}}\right] d x d t\right) d \rho .
\end{aligned}
$$

In (3.10) we have denoted by $\nabla_{x, t} \Gamma$ the total gradient of $\Gamma$, i.e., the $(n+1)$ dimensional vector $\left(\nabla_{y} \Gamma, \nabla_{z} \Gamma, D_{t} \Gamma\right)$. Also, with abuse of notation we have denoted by $\Gamma=\chi \rho^{-\gamma}$ the set $\psi_{\rho}\left(x_{0}, t_{0}\right)$. We recall that we have established (3.9) for a.e. $r>0$. At this point we make the change of variable $\sigma=\chi \rho^{-\gamma}$ in 
the 1.h.s. of (3.10), and then use Federer's co-area formula [Fe, Theorem 3.2.12, p. 249] obtaining

$$
\int_{0}^{r} \rho^{\gamma}\left(\int_{\Gamma=\chi / \rho^{\gamma}} u \frac{A \nabla_{y} \Gamma \cdot \nabla_{y} \Gamma}{\left|\nabla_{x, t} \Gamma\right|} d H_{n}\right) \frac{d \rho}{\rho}=\frac{\chi}{\gamma} \int_{\Omega_{r}\left(x_{0}, t_{0}\right)} u \frac{A \nabla_{y} \Gamma \cdot \nabla_{y} \Gamma}{\Gamma^{2}} d x d t .
$$

Replacing (3.11) in (3.10) finally gives

Theorem 3.1. Let $u \in C^{\infty}\left(\mathbf{R}^{n+1}\right),\left(x_{0}, t_{0}\right) \in \mathbf{R}^{n+1}$. Then we have

$$
\begin{aligned}
& -\int_{\partial \Omega_{r}\left(x_{0}, t_{0}\right)} u(x, t) A\left(\nabla_{y} \Gamma\left(x_{0}, t_{0} ; x, t\right)\right) \cdot \vec{N}_{y} d H_{n} \\
& =u\left(x_{0}, t_{0}\right)+\int_{\Omega_{r}\left(x_{0}, t_{0}\right)} u(x, t) L u(x, t)\left[\Gamma\left(x_{0}, t_{0} ; x, t\right)-\frac{\chi}{r^{\gamma}}\right] d x d t,
\end{aligned}
$$

for a.e. $r>0$. Moreover, for every $r>0$ we have

$$
\begin{aligned}
& \frac{\chi}{r^{\gamma}} \int_{\Omega_{r}\left(x_{0}, t_{0}\right)} u(x, t) \frac{A\left(\nabla_{y} \Gamma\left(x_{0}, t_{0} ; x, t\right)\right) \cdot \nabla_{y} \Gamma\left(x_{0}, t_{0} ; x, t\right)}{\Gamma^{2}\left(x_{0}, t_{0} ; x, t\right)} d x d t \\
& \quad=u\left(x_{0}, t_{0}\right)+\frac{\gamma}{r^{\gamma}} \int_{0}^{r} \rho^{\gamma}\left(\int_{\Omega_{\rho}\left(x_{0}, t_{0}\right)} L u(x, t)\left[\Gamma\left(x_{0}, t_{0} ; x, t\right)-\frac{\chi}{\rho^{\gamma}}\right] d x d t\right) \frac{d \rho}{\rho} .
\end{aligned}
$$

We stress the absence of the $\nabla_{z}$-part of the gradient of $\Gamma$ in the 1.h.s. of (3.13), a fact which reflects the degenerate nature of the operator $L$ in (1.13). Theorem 3.1 generalizes to the degenerate setting of this paper previous results in [GL1] for parabolic equations. We note that if $u$ is a solution of (1.13), then formula (3.13) gives (1.21). As previously pointed out, (1.21) is unsuitable for obtaining from it Harnack inequality since the kernel $\left(A\left(\nabla_{y} \Gamma\right) \cdot \nabla_{y} \Gamma\right) / \Gamma^{2}$ is quite singular.

Our next task is to obtain a family of representation formulas for $C^{\infty}$ functions on $\mathbf{R}^{n+1}$ which are well behaved from the viewpoint of Harnack inequality. The following lemma, besides being useful in the proof of Theorem 3.2 below, seems to have an independent interest.

Lemma 3.1. Let $u \in C^{\infty}\left(\mathbf{R}^{n+1}\right)$ and for $\left(x_{0}, t_{0}\right) \in \mathbf{R}^{n+1}$ and $r>0$ let us define (see (3.13))

$$
u_{r}\left(x_{0}, t_{0}\right)=\frac{\chi}{r^{\gamma}} \int_{\Omega_{r}\left(x_{0}, t_{0}\right)} u(x, t) \frac{A\left(\nabla_{y} \Gamma\left(x_{0}, t_{0} ; x, t\right)\right) \nabla_{y} \Gamma\left(x_{0}, t_{0} ; x, t\right)}{\Gamma^{2}\left(x_{0}, t_{0} ; x, t\right)} d x d t .
$$

Then

$$
\frac{d}{d r} u_{r}\left(x_{0}, t_{0}\right)=\frac{\gamma \chi}{r^{\gamma+1}} \int_{\Omega_{r}\left(x_{0}, t_{0}\right)} L u(x, t) \ln \left[\frac{r^{\gamma}}{\chi} \Gamma\left(x_{0}, t_{0} ; x, t\right)\right] d x d t
$$


Proof. By (3.13) in Theorem 3.1 we have

$$
\begin{aligned}
\frac{d}{d r} u_{r}\left(x_{0}, t_{0}\right)= & -\gamma^{2} r^{-\gamma-1} \int_{0}^{r} \rho^{\gamma-1}\left(\int_{\Omega_{\rho}\left(x_{0}, t_{0}\right)} L u\left[\Gamma-\frac{\chi}{\rho^{\gamma}}\right] d x d t\right) d \rho \\
& +\gamma r^{-1} \int_{\Omega_{r}\left(x_{0}, t_{0}\right)} L u\left[\Gamma-\frac{\chi}{r^{\gamma}}\right] d x d t .
\end{aligned}
$$

Using Fubini's theorem we can exchange the order of integration in the first term on the r.h.s. of (3.16) obtaining after an integration in the $\rho$-variable

$$
\begin{aligned}
\frac{d}{d r} u_{r}\left(x_{0}, t_{0}\right)= & -\gamma^{2} r^{-\gamma-1} \int_{\Omega_{r}\left(x_{0}, t_{0}\right)} L u\left[\frac{\Gamma \rho^{\gamma}}{\gamma}-\chi \ln \rho\right]_{\rho=(\chi / \Gamma)^{1 / \gamma}}^{\rho=r} d x d t \\
& +\frac{\gamma}{r} \int_{\Omega_{r}\left(x_{0}, t_{0}\right)} L u\left[\Gamma-\frac{\chi}{r^{\gamma}}\right] d x d t .
\end{aligned}
$$

A simple computation now yields (3.15) from (3.17). As a consequence of Lemma 3.1 we have the following monotonicity property of Kolmogorov averages.

Corollary 3.1. Let $u \in C^{\infty}\left(\mathbf{R}^{n+1}\right)$ and suppose that $L u \geq 0$. Then for every $\left(x_{0}, t_{0}\right) \in \mathbf{R}^{n+1}, 0<r<R$, and $\theta \in(0,1)$, there exists a constant $C=$ $C(\gamma, \chi, \theta)>0$ such that

$$
u_{R}\left(x_{0}, t_{0}\right)-u_{r}\left(x_{0}, t_{0}\right) \geq C\left(\frac{1}{r^{\gamma}}-\frac{1}{R^{\gamma}}\right) \int_{\Omega_{\theta r}\left(x_{0}, t_{0}\right)} L u(x, t) d x d t .
$$

Proof. (3.15) gives

$$
\begin{aligned}
& u_{R}\left(x_{0}, t_{0}\right)-u_{r}\left(x_{0}, t_{0}\right)=\int_{r}^{R} \frac{d}{d \rho} u_{\rho}\left(x_{0}, t_{0}\right) d \rho \\
& \quad=\gamma \chi \int_{r}^{R} \rho^{-\gamma-1}\left(\int_{\Omega_{\rho}\left(x_{0}, t_{0}\right)} L u \ln \left[\frac{\rho^{\gamma} \Gamma}{\chi}\right] d x d t\right) d \rho \\
& \quad \geq \gamma \chi \int_{\Omega_{\theta r}\left(x_{0}, t_{0}\right)} L u \ln \left[\frac{r^{\gamma}}{\chi} \Gamma\right] d x d t \int_{r}^{R} \rho^{-\gamma-1} d \rho .
\end{aligned}
$$

Observing now that on the set $\Omega_{\theta r}\left(x_{0}, t_{0}\right)$ we have $r^{\gamma} \Gamma / \chi>1 / \theta^{\gamma},(3.18)$ easily follows from (3.19) with $C=(\chi \gamma) \ln (1 / \theta)$.

Let now $u \in C^{\infty}\left(\mathbf{R}^{n+1}\right)$. If $h \in \mathbf{N}$ we define a function $u_{h} \in C^{\infty}\left(\mathbf{R}^{n+h+1}\right)$ by setting

$$
u_{h}(x, w, t)=u(x, t), \quad w \in \mathbf{R}^{h} .
$$

If $L_{h}$ denotes the operator defined in (1.23) we obviously have for every $(x, t) \in \mathbf{R}^{n+1}, w \in \mathbf{R}^{h}$,

$$
L_{h} u_{h}(x, w, t)=L u(x, t) .
$$


We remark explicitly that denoting by $I_{h}$ the identity matrix in $\mathbf{R}^{h}$, and by $0_{p, q}$ the $p \times q$ zero matrix, then $L_{h}$ can be written as follows

$$
L_{h} v=\operatorname{div}_{(y, w)}\left(A_{h} \nabla_{(y, w)} v\right)-B_{h}\left(\begin{array}{c}
y \\
w
\end{array}\right) \cdot \nabla_{(z, w)} v-D_{t} v,
$$

where we have set

$$
A_{h}=\left[\begin{array}{cc}
A & 0_{k, h} \\
0_{h, k} & I_{h}
\end{array}\right], \quad B_{h}=\left[\begin{array}{ll}
B & 0_{l, h}
\end{array}\right] .
$$

Since $B_{h} A_{h} B_{h}^{T}=B A B^{T}=C^{-1}$, we see that (3.21) defines in $\mathbf{R}^{n+h+1}$ an operator of the same type of $L$ in (1.13). Since

$$
A_{h}^{-1}=\left[\begin{array}{cc}
A^{-1} & 0_{k, l} \\
0_{h, k} & I_{h}
\end{array}\right]
$$

it is easily verified that, if we let

$$
\begin{gathered}
\left|\left(\begin{array}{c}
y \\
w
\end{array}\right)\right|_{A_{h}}^{2}=A_{h}^{-1}\left(\left[\begin{array}{l}
y \\
w
\end{array}\right]\right) \cdot\left[\begin{array}{l}
y \\
w
\end{array}\right], \\
|z|_{C_{h}}^{2}=C_{h} z \cdot z \quad \text { with } C_{h}=\left(B_{h} A_{h} B_{h}^{T}\right)^{-1},
\end{gathered}
$$

then

$$
\left|\left[\begin{array}{c}
y \\
w
\end{array}\right]\right|_{A_{h}}^{2}=|y|_{A}^{2}+|w|^{2}, \quad|z|_{C_{h}}^{2}=|z|_{C}^{2}
$$

where $|w|$ denotes the Euclidean length of $w \in \mathbf{R}^{h}$. These considerations and (1.15) allow us to conclude that if $\Gamma_{h}$ denotes the fundamental solution of $L_{h}$ in (3.21), then we have

$$
\Gamma_{h}\left(x_{0}, w_{0}, t_{0} ; x, w, t\right)=\Gamma\left(x_{0}, t_{0} ; x, t\right) K_{h}\left(w_{0}-w ; t_{0}-t\right)
$$

where $\Gamma$ is as in (1.15) and $K_{h}$ denotes the Gauss-Weierstrass kernel on $\mathbf{R}^{h+1}$, i.e.,

$$
K_{h}\left(w_{0}-w ; t_{0}-t\right)= \begin{cases}\left(4 \pi\left(t_{0}-t\right)\right)^{-h / 2} \exp \left[-\left|w_{0}-w\right|^{2} / 4\left(t_{0}-t\right)\right], & t_{0}>t, \\ 0, & t_{0} \leq t .\end{cases}
$$

At this point we define for $r>0$ in analogy with (1.22)

$$
\begin{aligned}
& E_{r}^{(h)}\left(x_{0}, w_{0}, t_{0} ; x, w, t\right) \\
& \quad=\frac{\chi h}{r^{\gamma_{h}}} \frac{A_{h}\left(\nabla_{(y, w)} \Gamma_{h}\left(x_{0}, w_{0}, t_{0} ; x, w, t\right)\right) \cdot \nabla_{(y, w)} \Gamma_{H}\left(x_{0}, w_{0}, t_{0} ; x, w, t\right)}{\Gamma_{h}^{2}\left(x_{0}, w_{0}, t_{0} ; x, w, t\right)}
\end{aligned}
$$


where $\chi_{h}$ and $\gamma_{h}$ are given by (1.26). In virtue of (3.23) and by the definition of $A_{h}$ we obtain

$$
\begin{array}{r}
E_{r}^{(h)}\left(x_{0}, w_{0}, t_{0}, x, w, t\right)=\frac{\chi_{h}}{r^{\gamma_{h}}}\left[\frac{A\left(\nabla_{y} \Gamma\left(x_{0}, t_{0} ; x, t\right)\right) \cdot \nabla_{y} \Gamma\left(x_{0}, t_{0} ; x, t\right)}{\Gamma^{2}\left(x_{0}, t_{0} ; x, t\right)}\right. \\
\left.+\frac{\left|\nabla_{w} K_{h}\left(w_{0}-w\right)\right|^{2}}{K_{h}^{2}\left(w_{0}-w\right)}\right] \\
=\frac{\chi_{h}}{r^{\gamma_{h}}}\left[\frac{A\left(\nabla_{y} \Gamma\left(x_{0}, t_{0} ; x, t\right)\right) \cdot \nabla_{y} \Gamma\left(x_{0}, t_{0} ; x, t\right)}{\Gamma^{2}\left(x_{0}, t_{0} ; x, t\right)}+\frac{\left|w_{0}-w\right|^{2}}{4\left(t_{0}-t\right)^{2}}\right] .
\end{array}
$$

The last equality in (3.23) follows by a direct computation in (3.24).

If $u_{h}$ is as in (3.20) and $r>0$ we denote by $\left(u_{h}\right)_{r}$ the $(n+h+1)$-dimensional Kolmogorov average of $u_{h}$ defined analogously to (3.14), i.e.,

$\left(u_{h}\right)_{r}\left(x_{0}, w_{0}, t_{0}\right)=\int_{\widehat{\Omega}_{r}\left(x_{0}, w_{0}, t_{0}\right)} u(x, w, t) E_{r}^{(h)}\left(x_{0}, w_{0}, t_{0} ; x, w, t\right) d x d w d t$,

where $E_{r}^{(h)}$ is defined by (3.25) and we have let

$$
\widehat{\Omega}_{r}\left(x_{0}, w_{0}, t_{0}\right)=\left\{(x, w, t) \in \mathbf{R}^{n+h+1} \mid \Gamma_{h}\left(x_{0}, w_{0}, t_{0} ; x, w, t\right)>\chi_{h} / r^{\gamma_{h}}\right\} .
$$

Using now (3.23), (3.26), and Fubini's theorem we obtain

$$
\begin{aligned}
\left(u_{h}\right)_{r}( & \left.x_{0}, w_{0}, t_{0}\right)=\frac{\chi_{h}}{r^{\gamma_{h}}} \int_{\widehat{\Omega}_{r}\left(x_{0}, w_{0}, t_{0}\right)} u\left[\frac{A\left(\nabla_{y} \Gamma\right) \cdot \nabla_{y} \Gamma}{\Gamma^{2}}+\frac{\left|w_{0}-w\right|^{2}}{4\left(t_{0}-t\right)^{2}}\right] d x d w d t \\
= & \frac{\chi_{h}}{r^{\gamma_{h}}} \int_{\Phi>\chi r^{-\gamma_{h}}} u \frac{A\left(\nabla_{y} \Gamma\right) \cdot \nabla_{y} \Gamma}{\Gamma^{2}}\left(\int_{\left|w_{0}-w\right|<R_{r}\left(t_{0}-t\right)} d w\right) d x d t \\
& +\frac{\chi_{h}}{r^{\gamma_{h}}} \int_{\Phi>\chi r^{-\gamma_{h}}} \frac{u}{4\left(t_{0}-t\right)^{2}}\left(\int_{\left|w_{0}-w\right|<R_{r}\left(t_{0}-t\right)}\left|w_{0}-w\right|^{2} d w\right) d x d t,
\end{aligned}
$$

where we have set

$$
\begin{aligned}
& \Phi\left(x_{0}, t_{0} ; x, t\right)=\left(t_{0}-t\right)^{-h / 2} \Gamma\left(x_{0}, t_{0} ; x, t\right) \\
& R_{r}\left(t_{0}-t\right)^{2}=r\left(t_{0}-t\right) \ln \left[\frac{r^{\gamma_{h}} \Phi\left(x_{0}, t_{0} ; x, t\right)}{\chi}\right] .
\end{aligned}
$$

If we denote by $\omega_{h}$ the volume of the $h$-dimensional unit ball we have

$$
\begin{aligned}
\int_{\left|w_{0}-w\right|<R_{r}\left(t_{0}-t\right)} d w & =\omega_{h} R_{r}\left(t_{0}-t\right)^{h} \\
\int_{\left|w_{0}-w\right|<R_{r}\left(t_{0}-t\right)}\left|w_{0}-w\right|^{2} d w & =\frac{h}{h+2} \omega_{h} R_{r}\left(t_{0}-t\right)^{h+2} .
\end{aligned}
$$


Recalling (1.25), we finally obtain from (3.29)

$$
\left(u_{h}\right)_{r}\left(x_{0}, w_{0}, t_{0}\right)=\int_{\Omega_{r}^{(h)}\left(x_{0}, t_{0}\right)} u(x, t) E_{r}^{(h)}\left(x_{0}, t_{0} ; x, t\right) d x d t,
$$

where we have defined for $R_{r}\left(t_{0}-t\right)$ as in (3.31)

$$
\begin{array}{r}
E_{r}^{(h)}\left(x_{0}, t_{0} ; x, t\right)=\frac{\omega_{h} \chi_{h}}{r^{\gamma_{h}}} R_{r}\left(t_{0}-t\right)^{h}\left[\frac{A\left(\nabla_{y} \Gamma\left(x_{0}, t_{0} ; x, t\right)\right) \cdot \nabla_{y} \Gamma\left(x_{0}, t_{0} ; x, t\right)}{\Gamma^{2}\left(x_{0}, t_{0} ; x, t\right)}\right. \\
\left.+\frac{h}{h+2} \frac{R_{r}\left(t_{0}-t\right)^{2}}{\left(t_{0}-t\right)^{2}}\right] .
\end{array}
$$

We are now ready to use Lemma 3.1. Integrating (3.15) in $r$ and noting that $\lim _{r \rightarrow 0+} u_{r}\left(x_{0}, t_{0}\right)=u\left(x_{0}, t_{0}\right)$ we obtain

$$
u_{r}\left(x_{0}, t_{0}\right)=u\left(x_{0}, t_{0}\right)+\gamma \chi \int_{0}^{r} \rho^{-\gamma}\left(\int_{\Omega_{\rho}\left(x_{0}, t_{0}\right)} L u \ln \left[\frac{\rho^{\gamma}}{\chi} \Gamma\right] d x d t\right) \frac{d \rho}{\rho} .
$$

We apply (3.34) to deduce a $(n+k+1)$-dimensional representation formula for the function $u_{h}$ in (3.20). Writing the $(n+k+1)$-dimensional version of (3.34) for $u_{h}$ and using (3.32) we end up with

$$
\begin{aligned}
\int_{\Omega_{r}^{(h)}\left(x_{0}, t_{0}\right)} u(x, t) E_{r}^{(h)}\left(x_{0}, t_{0} ; x, t\right) d x d t=u\left(x_{0}, t_{0}\right) \\
+\gamma_{h} \chi_{h} \int_{0}^{r} \rho^{-\gamma_{h}}\left(\int_{\widehat{\Omega}_{\rho}\left(x_{0}, w_{0}, t_{0}\right)} L_{h} u_{h}(x, w, t)\right. \\
\left.\cdot \ln \left[\frac{\rho^{\gamma_{h}} \Gamma_{h}\left(x_{0}, w_{0}, t_{0} ; x, w, t\right)}{\chi_{h}}\right] d x d w d t\right) \frac{d \rho}{\rho} .
\end{aligned}
$$

In the integral in the r.h.s of (3.35) $\widehat{\Omega}_{\rho}\left(x_{0}, w_{0}, t_{0}\right)$ is as in (3.28). Now we observe that because of $(1.26),(3.23),(3.30),(3.31)$ we have

$$
\ln \left[\frac{\rho^{\gamma_{h}} \Gamma_{h}}{\chi_{h}}\right]=\frac{1}{4\left(t_{0}-t\right)}\left[R_{\rho}\left(t_{0}-t\right)^{2}-\left|w_{0}-w\right|^{2}\right] .
$$

Using (3.36), the fact that $L_{h} u_{h}=L u$, and a splitting of the integrals similar to that performed in (3.29), we finally obtain from (3.35):

Theorem 3.2. Let $u \in C^{\infty}\left(\mathbf{R}^{n+1}\right)$ and let $\left(x_{0}, t_{0}\right) \in \mathbf{R}^{n+1}$. For any $h \in \mathbf{N}$ we have for every $r>0$

$$
\begin{aligned}
& \int_{\Omega_{r}^{(h)}\left(x_{0}, t_{0}\right)} u(x, t) E_{r}^{(h)}\left(x_{0}, t_{0} ; x, t\right) d x d t \\
&= u\left(x_{0}, t_{0}\right)+\frac{2 \gamma+h}{h+2} \omega_{h}(4 \pi)^{-h / 2} \chi \\
& \cdot \int_{0}^{r} \rho^{-\gamma_{h}}\left(\int_{\Omega_{\rho}^{(h)}\left(x_{0}, t_{0}\right)} L u(x, t) \frac{R_{\rho}\left(t_{0}-t\right)^{h+2}}{4\left(t_{0}-t\right)} d x d t\right) \frac{d \rho}{\rho},
\end{aligned}
$$


where $\gamma_{h}, E_{r}^{(h)}$, and $R_{r}\left(t_{0}-t\right)$ are respectively given by (1.26), (3.33) and (3.31). In particular, if $u$ is a solution of (1.13) in the set $\Omega_{r}^{(h)}\left(x_{0}, t_{0}\right)$ we have

$$
u\left(x_{0}, t_{0}\right)=\int_{\Omega_{r}^{(h)}\left(x_{0}, t_{0}\right)} u(x, t) E_{r}^{(h)}\left(x_{0}, t_{0} ; x, t\right) d x d t .
$$

\section{Proof of Theorem 1.1}

Theorem 3.2 above is the ad hoc tool for proving the uniform Harnack inequality expressed in Theorem 1.1. Our proof will closely imitate, via the three-balls lemma, the classical proof for harmonic functions. One piece of information is, however, still missing, namely, the boundedness of the kernel $E_{r}^{(h)}$ appearing in (3.38). In what follows we let $u$ be a nonnegative solution of (1.13) in the set $\Omega_{4 r}^{(h)}\left(x_{0}, t_{0}\right)$ as in the statement of Theorem 1.1. By (3.38), the three-balls lemma and the fact that $u \geq 0$ we obtain

$$
\begin{aligned}
u\left(x_{0}, t_{0}\right) & =\int_{\Omega_{3 r}^{(h)}\left(x_{0}, t_{0}\right)} u(\xi, \tau) E_{3 r}^{(h)}\left(x_{0}, t_{0} ; \xi, \tau\right) d \xi d \tau \\
& \geq \int_{\Omega_{\delta r}^{(h)}(x, t)} u(\xi, \tau) \frac{E_{3 r}^{(h)}\left(x_{0}, t_{0} ; \xi, \tau\right)}{E_{\delta r}^{(h)}(x, t ; \xi, \tau)} E_{\delta r}^{(h)}(x, t ; \xi, \tau) d \xi d \tau .
\end{aligned}
$$

Suppose for a moment we could prove that for $\varepsilon \in(0,1)$ fixed, there exists a $C=C(\varepsilon, n, h, L)>0$ such that for any $(x, t) \in \Omega_{r}^{(h)}\left(x_{0}, t_{0}\right)$ for which $t_{0}-t \geq \varepsilon r$

$$
\begin{gathered}
\sup _{(\xi, \tau) \in \Omega_{\delta r}^{(h)}(x, t)} E_{\delta r}^{(h)}(x, t ; \xi, \tau) \leq C r^{-(\gamma+1)}, \\
\inf _{(\xi, \tau) \in \Omega_{\delta r}^{(h)}(x, t)} E_{3 r}^{(h)}\left(x_{0}, t_{0} ; \xi, \tau\right) \geq C r^{-(\gamma+1)} .
\end{gathered}
$$

Replacing (4.2), (4.3) into (4.1) we would then obtain(1.27).

In order to complete the proof of Theorem 1.1 we are therefore left with proving (4.2), (4.3). We begin with the easy part, namely, (4.3). Using again the three-balls lemma, and by (3.33), we obtain for $t_{0}-t \geq \varepsilon r$

$$
\begin{aligned}
& \inf _{(\xi, \tau) \in \Omega_{\delta r}^{(h)}(x, t)} E_{3 r}^{(h)}\left(x_{0}, t_{0} ; \xi, \tau\right) \geq \inf _{\substack{(\xi, \tau) \in \Omega_{2 r}^{(h)}\left(x_{0}, t_{0}\right)\\
}} E_{3 r}^{(h)}\left(x_{0}, t_{0} ; \xi, \tau\right) \\
& \geq \frac{h}{h+2} \frac{\omega_{h} \chi_{h}}{(3 r)^{\gamma_{h}}} \inf _{\substack{(\xi, \tau) \in \Omega_{2}^{(h)}\left(x_{0}, t_{0}\right) \\
t_{0}-\tau \geq \varepsilon r}} \frac{\left[R_{3 r}\left(t_{0}-r\right)^{2}\right]^{h / 2+1}}{\left(t_{0}-\tau\right)^{2}} .
\end{aligned}
$$

Recalling now the definitions (1.25), (3.30), (3.31) we have on $\Omega_{2 r}^{(h)}\left(x_{0}, t_{0}\right)$, and for $t_{0}-\tau \geq \varepsilon r$

$$
\begin{aligned}
\frac{\left[R_{3 r}\left(t_{0}-\tau\right)^{2}\right]^{h / 2+1}}{\left(t_{0}-\tau\right)^{2}} & =4^{h / 2+1}\left(t_{0}-\tau\right)^{h / 2-1} \ln \left[\frac{(3 r)^{\gamma / h} \Phi\left(x_{0}, t_{0} ; \xi, \tau\right)}{\chi}\right] \\
& \geq \gamma_{h} 4^{h / 2+1} \ln \left(\frac{3}{2}\right) \varepsilon^{h / 2-1} r^{h / 2-1},
\end{aligned}
$$


provided that $h \geq 2$. Substituting (4.5) into (4.4) yields (4.3). We now examine (4.2). The first step is to estimate $\left(A\left(\nabla_{\eta} \Gamma\right) \cdot \nabla_{\eta} \Gamma\right) / \Gamma^{2}$ in the set $\Omega_{\delta r}^{(h)}(x, t)$. By (1.25) and (3.30) we see that if $x=(y, z)$, then $\Omega_{\delta r}^{(h)}(x, t)$ is the set of all points $(\xi, \tau)=(\eta, \zeta, \tau) \in \mathbf{R}^{n+1}$ such that $0<t-\tau<\delta r$ and

(4.6) $\frac{1}{4}|y-\eta|_{A}^{2}+\frac{3}{(t-\tau)^{2}}\left|\zeta-z+\frac{(t-\tau)}{2} B(y+\eta)\right|_{C}^{2}<\gamma_{h}(t-\tau) \ln \left(\frac{\delta r}{t-\tau}\right)$.

From (1.15) we obtain

$$
\frac{\nabla_{\eta} \Gamma(x, t ; \xi, \tau)}{\Gamma(x, t ; \xi, \tau)}=-\frac{(\eta-y)^{T} A^{-1}}{2(t-\tau)}-\frac{3}{(t-\tau)^{2}}\left(\zeta-z+\frac{(t-\tau)}{2} B(y+\eta)\right)^{T} C B .
$$

Assumption (1.14) implies the existence of two numbers $\lambda, \mu>0$ such that

$$
\begin{cases}\lambda|y|^{2} \leq|y|_{A}^{2} \leq \lambda^{-1}|y|^{2}, & y \in \mathbf{R}^{k}, \\ \mu|z|^{2} \leq|z|_{C}^{2} \leq \mu^{-1}|z|^{2}, & z \in \mathbf{R}^{l},\end{cases}
$$

where $|y|$ and $|z|$ denote Euclidean norms. (4.7) and (4.8) imply

$$
\frac{A\left(\nabla_{\eta} \Gamma\right) \cdot \nabla_{\eta} \Gamma}{\Gamma^{2}} \leq C\left\{\frac{|\eta-y|_{A}^{2}}{(t-\tau)^{2}}+\frac{|\zeta-z+((t-\tau) / 2) B(y+\eta)|_{C}^{2}}{(t-\tau)^{4}}\right\}
$$

for a constant $C=C(n, L)>0$. Using, at this point, (4.6) in (4.9) yields for a $C=C(n, h, L)>0$

$$
\frac{A\left(\nabla_{\eta} \Gamma\right) \cdot \nabla_{\eta} \Gamma}{\Gamma^{2}} \leq C \frac{1}{t-\tau} \ln \left(\frac{\delta r}{t-\tau}\right) \quad \text { in } \Omega_{\delta r}^{(h)}(x, t) .
$$

Recalling (3.33) we see that in order to prove (4.2) we must estimate $R_{\delta r}(t-\tau)=$ $R_{\delta r}(x, t ; \xi, \tau)$ from above in $\Omega_{\delta r}^{(h)}(x, t)$. From (3.31) and (3.30) we have

$$
R_{\delta r}(t-\tau)^{2}=4(t-\tau) \ln \left[\frac{(\delta r)^{\gamma_{h}} \phi(x, t ; \xi, \tau)}{\chi}\right] \leq 4(t-\tau) \ln \left(\frac{C r}{t-\tau}\right)
$$

for a constant $C=C(\delta, h, \chi)>0$. In conclusion, see (3.33),

$$
\sup _{(\xi, \tau) \in \Omega_{\delta r}^{(h)}(z, t)} R_{\delta r}(t-\tau)^{h} \frac{A\left(\nabla_{\eta} \Gamma\right) \cdot \nabla_{\eta} \Gamma}{\Gamma^{2}} \leq C(t-\tau)^{h / 2-1}\left[\ln \left(\frac{C r}{t-\tau}\right)\right]^{h / 2+1}
$$

and analogously,

$$
\sup _{(\xi, \tau) \in \Omega_{\delta r}^{(h)}(x, t)} \frac{R_{\delta r}(t-\tau)^{h-2}}{(t-\tau)^{2}} \leq C(t-\tau)^{h / 2-1}\left[\ln \left(\frac{C r}{t-\tau}\right)\right]^{h / 2+1} .
$$

In (4.11), (4.12) we have denoted by $C$ a positive constant depending on $\varepsilon$, $n, h, L$. Letting now $h>2$ in (4.11), (4.12), and recalling (3.33) once more, we finally obtain (4.2). This completes the proof of Theorem 1.1. 


\section{BIBLIOGRAPHY}

[C] C. Chandrasekhar, Stochastic problems in physics and astronomy, Rev. Modern Phys. 15 (1943), 1-89.

[FS] E. Fabes and D. W. Stroock, A new proof of Moser's parabolic Harnack inequality via the old ideas of Nash, Arch. Rational Mech. Anal. 96 (1986), 327-338.

[GL1] N. Garofalo and E. Lanconelli, Wiener's criterion for parabolic equations with variable coefficients and its consequences, Trans. Amer. Math. Soc. 308 (1988), 811-836.

[GL2] __ Asymptotic behavior of fundamental solutions and potential theory of parabolic operators with variable coefficients, Math. Ann. 283 (1989), 211-240.

[H] L. Hörmander, Hypoelliptic second order differential equations, Acta Math. 119 (1967), 147 171.

[I] A. M. I''in, On a class of ultraparabolic equations, Dokl. Akad. Nauk SSSR 159 (1964), 1214-1217.

[JS] D. Jerison and A., Sanchez-Calle, Estimates for the heat kernel for a sum of squares of vector fields, Indiana Univ. Math. J. 35 (1986), 835-854.

[K] A. Kolmogorov, Zufällige Bewegungen, Ann. of Math. 35 (1934), 116-117.

[Ku] L. P. Kupcov, Mean value theorem and a maximum principle for Kolmogorov's equation, Math. Notes 15 (1974), 280-286.

[KS1] S. Kusuoka and D. W. Stroock, Applications of Malliavin calculus. III, J. Fac. Sci. Univ. Tokyo Sect. IA Math. 34 (1987), 391-442.

[KS2] __ Long time estimates for the heat kernel associated with a uniformly subelliptic symmetric second order operator, Ann. of Math. (2) 127 (1988), 165-189.

[N] J. Nash, Continuity of solutions of parabolic and elliptic equations, Amer. J. Math. 80 (1958), 931-954.

[S] A. Sanchez-Calle, Fundamental solutions and geometry of the sum of squares of vector fields, Invent. Math. 78 (1984), 143-160.

[SV] D. W. Stroock and S. R. S. Varadhan, Multidimensional diffusion processes, Springer-Verlag, Berlin, Heidelberg, New York, 1979.

[W.] M. Weber, The fundamental solution of a degenerate partial differential equation of parabolic type, Trans. Amer. Math. Soc. 71 (1951), 24-37.

Department of Mathematics, Northwestern University, Evanston, Illinois 60208

Dipartimento di Matematica, Università di Bologna, 40126 Bologna, Italy (Current address of Ermanno Lanconelli)

Current address (Nicola Garofalo): Department of Mathematics, Purdue University, West Lafayette, Indiana 47907 